\title{
The Brentano illusion influences goal-directed movements of the left and right hand to the same extent
}

\author{
Denise D. J. de Grave · Eli Brenner · Jeroen B. J. Smeets
}

Received: 6 November 2007 / Accepted: 29 October 2008 / Published online: 15 November 2008

(C) The Author(s) 2008. This article is published with open access at Springerlink.com

\begin{abstract}
Recently, Gonzalez et al. (J Neurophys 95:3496-3501, 2006) reported that movements with the left hand are more susceptible to visual size illusions than are those with the right hand. We hypothesized that this might be because proprioceptive information about the position of the left hand is less precise. If so, the difference between the hands should become clearer when vision of the hand is removed so that subjects can only rely on proprioception to locate their hand. We tested whether this was so by letting right-handed subjects make open-loop pointing movements within an illusory context with and without vision of their hand. On average, the illusion influenced the left and the right hand to the same extent, irrespective of the visibility of the hand. There were some systematic differences between the hands within certain regions of space, but these were not consistent across subjects. We conclude that there is no fundamental difference between the hands in susceptibility to the Brentano illusion.
\end{abstract}

Keywords Visual illusions · Perception-action .

Handedness $\cdot$ Proprioception $\cdot$ Information

\section{Introduction}

When investigating the effect of visual illusions on visuomotor performance, tasks are normally performed with the dominant hand. For most people this is the right one. Gonzalez et al. (2006) examined how visual illusions affect

D. D. J. de Grave $(\bowtie)$ · E. Brenner · J. B. J. Smeets

Research Institute MOVE, Faculty of Human Movement Sciences,

VU University Amsterdam, Van der Boechorststraat 9,

1081 BT Amsterdam, The Netherlands

e-mail: d.degrave@fbw.vu.nl grasping movements with the left and the right hand in both left- and right-handed subjects. They showed subjects small objects, which they had to pick up from within an illusory context. The illusion affected maximum grip aperture more when grasping movements were performed with the left hand than when they were performed with the right hand, irrespective of the subject's handedness.

Although this seems a rather strange result, one may not simply expect left-handed subjects to behave in a mirrorsymmetric manner to right-handed ones. When left and right-handed subjects are asked to grasp small objects with a precision grip, right-handed subjects prefer to use their dominant hand whereas left-handed subjects use their right hand about half of the time (Gonzalez et al. 2007; see also Stins et al. 2001). Several other studies have also shown differences in performance between the arms (reviewed in Goble and Brown 2008). Important for the present study is the finding of Van Beers et al. (1998), who demonstrated that the non-visual (proprioceptive) information about the position of the hand is not only more precise for the right hand for right-handed subjects, but also for left-handed ones.

The asymmetry in proprioceptive accuracy is the basis of a possible explanation for the larger effect of illusions on the left hand. A lower precision in proprioception for the left than the right hand means that egocentric information about the location of the left hand is less precise than egocentric information about the location of the right hand. As subjects are likely to rely less on imprecise information (van Beers et al. 2002) they will rely more on other sources of information such as movement distance when using their left hand, and therefore be more strongly influenced by illusions of length (de Grave et al. 2004).

Whether this can account for the influence on grip aperture in the grasping study of Gonzalez et al. (2006) depends on how one thinks that the effect of context items on grip 
aperture arises. The effect of the illusion implies that we must reject the idea (Smeets and Brenner 1999) that egocentric information about the positions of the contact points is the only information that is used. A first option is that allocentric information about the object's size, which is influenced by the illusion, is also used (Jeannerod 1981). Alternatively, elements in the surrounding context may be regarded as obstacles that subjects have to take care not to collide with (Biegstraaten et al. 2007; de Grave et al. 2005; Haffenden and Goodale 2000). Both giving size more consideration and taking more care to avoid obstacles when moving with the left hand, would be consistent with the effect of the illusory context having more influence on the less precise left hand than on the more precise right hand.

If the difference in proprioceptive precision underlies the difference in illusion effects between the hands, the latter difference should increase when subjects rely more on proprioception rather than vision to localize the hand. In this study we investigate whether this is so by comparing the effect of an illusion when visibility of the hand is removed with that when the hand remains visible. When the hand is invisible, subjects cannot rely on visual information regarding the position of their hand, but have to use proprioceptive information, which is less precise for the left hand. Since grasping is known to be influenced by factors such as posture (Cuijpers et al. 2004) and the consistency of haptic feedback (Cuijpers et al. 2008), we chose to use a simpler, pointing task on a graphics tablet. We chose the Brentano illusion because it is known to affect pointing movements considerably, but still much less than it affects size judgments, so we can be sure that we will be able to detect small changes in the effect of the illusion.

\section{Materials and methods}

The methods are very similar to two conditions of an earlier experiment in which subjects had to move a pen to a target that disappeared as soon as the pen started to move (de Grave et al. 2004). In the first condition the hand was continuously visible, whereas in the second condition the hand was invisible throughout the experiment. The critical difference with the study of de Grave et al. (2004) is that subjects performed each condition twice: once with their left hand and once with their right hand.

\section{Subjects}

Ten subjects took part in this study. All subjects performed both conditions. They all had normal or corrected-to-normal vision and were right-handed according to the Edinburgh Handedness Inventory score (Oldfield 1971). One subject had a score of 80 ; the score for all the others was
100. The study was approved by the ethics committee of the Faculty of Human Movement Science.

Stimulus and apparatus

In both conditions the stimulus consisted of a black Brentano figure and a red target dot (diameter $0.2 \mathrm{~cm}$ ) on a white background. The two aligned horizontal shafts each were $8 \mathrm{~cm}$ in length. The length of the wings was $2.2 \mathrm{~cm}$. The inclination of the wings with respect to the shafts was $30^{\circ}$. Two configurations of the Brentano illusion were used: one with the wings-in on the left side (Fig. 1a) and one with the wings-out on the left side (Fig. 1b).

The target dot could appear either on one of the three arrowheads of the Brentano illusion (left, middle or right) or outside the figure. The target dot outside the figure was presented $8 \mathrm{~cm}$ below the middle arrowhead. One configuration of the figure and one target dot were presented on each trial.

The stimulus was projected on a projection surface above a graphic tablet (Fig. 1c). Subjects looked at the projected image via a semi-transparent mirror that was placed exactly half way between the projection surface and the tablet. They held a pen in their hand underneath the mirror. In the "hand visible" condition, bright lights beneath the mirror ensured that subjects could see their hand through the mirror at all times. For the "hand invisible" condition, these lights were switched off to ensure that subjects could not see their hand.

The size of the projected image was 1,024 by 768 pixels, with 1 pixel corresponding to about $0.5 \mathrm{~mm}$. Pointing positions were registered as the positions at which the tip of the pen stopped moving on the graphics tablet. This was defined as the position on the first sample of a 300-ms interval during which the tip of the pen had moved less than $1 \mathrm{~mm}$. Positions were sampled at $200 \mathrm{~Hz}$ with an accuracy of about $0.25 \mathrm{~mm}$.

\section{Procedure}

The two conditions were performed on different days, with a 4-week interval between them. All subjects performed the "hand visible" condition first. In each condition subjects performed four blocks of pointing trials; one for each configuration of the Brentano figure for each hand. Each block contained 400 trials. The order of the blocks was counterbalanced across subjects. Subjects were asked to bring the tip of the pen to the red target dot. The endpoint of each movement was the starting position of the next movement. On each trial the target appeared at a random location, with the chance of it appearing at the middle location being twice as large as the other locations. A movement between two locations was made at least 20 times in each block. Before the start of each condition subjects performed 15 practice trials with each hand. 
Fig. 1 The Brentano illusion with the wings-in configuration on the left side (a) and on the right side (b). The dots indicate the positions at which a target could appear (on one of the arrowheads or outside the illusion). Only one configuration was visible at a time. A schematic view of the apparatus (c). Subjects looked at the projected image via a semitransparent mirror. Underneath the mirror, they held a pen in their hand. In the hand-visible condition lights underneath the semitransparent mirror were turned on to enable subjects to see their hand through the mirror. For the hand invisible condition these lights were turned off. The possible movements in the stimulus divided into three types of movement (d): movements in which a large, medium and no illusion effect are expected. The symbols are used in Fig. 3

\section{a}

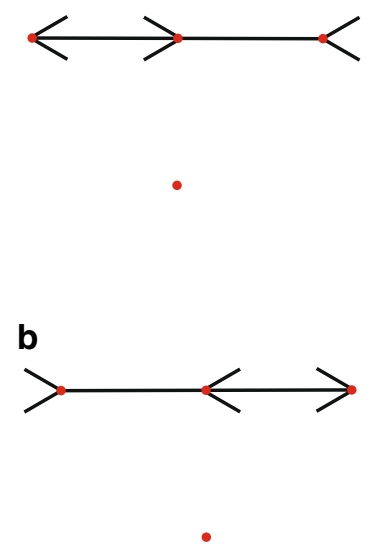

d

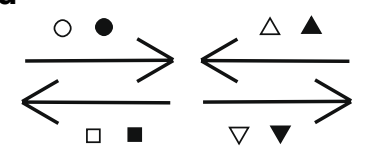

large
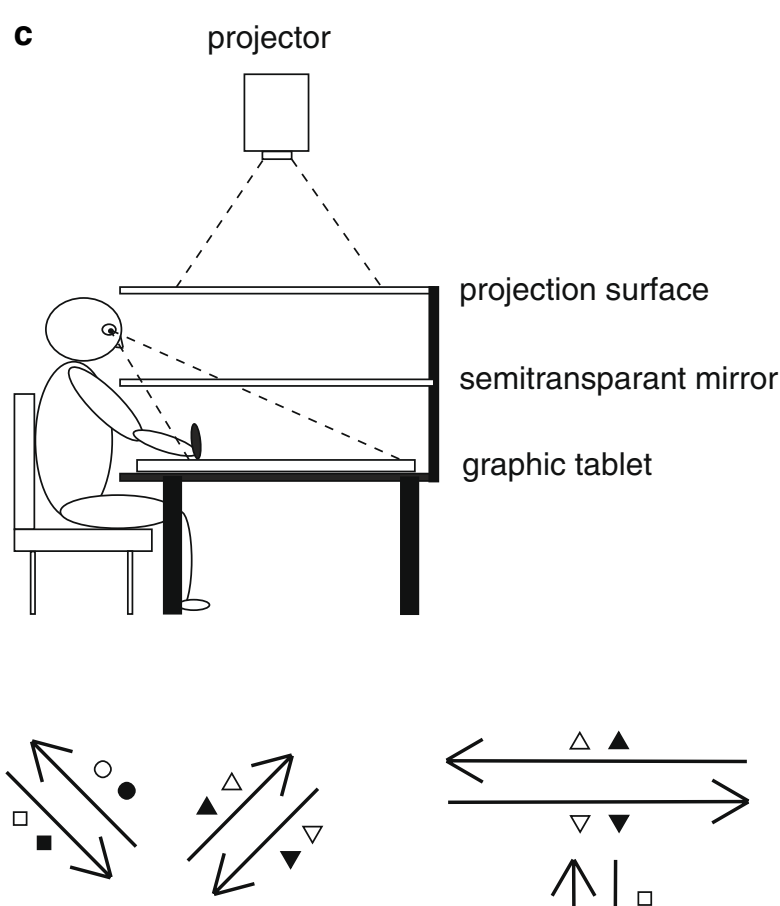

medium
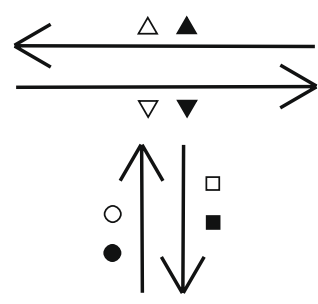

no

In both conditions the Brentano figure with the target dot was only visible before subjects started moving. As soon as subjects moved the pen, the stimulus disappeared from view. When the pen stopped again the Brentano figure reappeared with the target dot at a new position in the Brentano figure. In the "hand invisible" condition the Brentano figure always appeared at the same position on the screen. In the "hand visible" condition this would give the subjects information about their performance. Therefore in the latter condition, the computer aligned the arrowhead (or the position $8 \mathrm{~cm}$ below the middle arrowhead) to which the subject had just moved with the tip of the pen when the stimulus re-appeared, so that it seemed to the subjects as if they were always exactly on target. If the pen drifted outside the central part of the tablet, the stimulus reappeared at the middle of the tablet, and the following movement was discarded. On average, 34 trials of each subjects' 1,600 trials were discarded for this reason.

\section{Data analysis}

The quantitative comparison of the influence of the illusion is based on three types of movements, which differ in the expected effect of the illusion. In a previous study (de Grave et al. 2004), movements along the shaft of the Muller-Lyer illusion showed a large effect of the illusion. Movements perpendicular to the shaft and movements

between the outer arrowheads showed no effect of the illusion. Movements in a direction that formed an angle of $45^{\circ}$ with the shaft were mildly affected by the illusion. Thus, based on that study we divided the movements into three categories: movements in which a large effect of the illusion is expected (movements between the middle arrowhead and the outer arrowheads); movements in which a medium sized illusion effect is expected (movements between the outside location and the outer arrowheads) and finally movements in which no effect is expected (movements between the outer arrowheads and between the outside location and the middle arrowhead) (Fig. 1d).

To disregard any errors that are not related to the illusion, we determined the size of the illusion effect in the following way. We calculated the difference between the mean horizontal distances between the endpoints of pointing movements for the two configurations (wings-in on left, wings-out on left). We did so for each subject, performing hand and movement type. This difference in traversed distance between the two configurations was divided by the mean traversed distance when moving between the leftmost and rightmost arrowheads to correct for general tendencies to overestimate or underestimate the extent. The result is a measure of the size of the illusion, expressed as a percentage of the length of the movement along a shaft of the Brentano figure.

Statistical tests were all conducted among subjects. A repeated measures ANOVA with the factors visibility 
(hand visible, hand invisible), hand (left, right) and movement type (movements with a large, medium or no expected effect) was performed on our measure of the size of the illusion. Tukey post hoc tests were performed to see which levels of a factor differed. One sample $t$ tests were performed to check whether the illusion magnitude differed from zero for each visibility condition, each of the hands and each type of movement (i.e. to check whether there was any effect of the illusion at all).

To find out whether the left and the right hand differed in variability, standard deviations of the moved amplitudes in each visibility condition were calculated for each subject, performing hand and movement type. A repeated measures ANOVA was performed on the standard deviations with the factors visibility, hand and movement type.

\section{Results}

The illusion influenced pointing movements in which a large illusion effect is expected (movements between the middle arrowhead and the two outer arrowheads). This was the case for the left and the right hand, in both visibility conditions. The effect of the illusion was about $5 \%$ in the condition in which the hand was visible (Fig. 2a) and around $7 \%$ in the condition in which the hand was invisible (Fig. 2b). As expected, movements between the position outside the illusion and the outer arrowheads showed intermediate effects (about 2\% for hand visible; Fig. $2 \mathrm{a}$ and about 3\% for hand invisible; Fig. 2b). Movements perpendicular to the shaft and movements between the outer arrowheads showed no effect of the illusion (hand visible and hand invisible both less than $0.5 \%$ ).

The ANOVA on the illusion effects showed no difference in the size of the illusion between the hands $(F(1,9)=0.23, \quad p=0.64)$ or the visibility conditions $(F(1,9)=0.53, p=0.48)$. As already clearly visible in Fig. 2, the effect of the illusion did depend on the type of movement $(F(2,18)=32.76, p<0.001)$. No interactions were found. Post-hoc analysis showed that the effect of the illusion differed significantly between all three types of movement.

Figure 3 shows the average illusion effects for the individual subjects. The most conspicuous finding is that there are larger individual differences for movements in which the hand was invisible (open symbols) than for movements in which the hand was visible (filled symbols). Figure $3 \mathrm{a}$ shows movements between the outer arrowheads and the central arrow. For the hand-visible condition there is a remarkable consistency in the influence of the illusion between the hands: most filled symbols are near the unity line, meaning that although the size of the illusion effect differed between subjects, the effect was similar for both hands (correlation coefficient $r=0.71, p<0.001$ ). Movements between the outer arrowheads and between the outside location and the middle arrowhead, for which no effect (and thus no correlation) is expected, did not show any correlation (Fig. 3c, hand visible: $r=-0.09, p=0.56$ ). Movements between the outside location and the outer arrowheads, for which an intermediate illusion effect is expected, indeed show an intermediate correlation (Fig. 3b, hand visible: $r=0.25, p=0.11$ ). No significant correlations were found in the hand-invisible condition, despite the slightly (but not significantly) larger effect of the illusion.

We noticed when making Fig. 3a and $b$ that, for many subjects, movements on the left side of the stimulus (circles and squares) were on the opposite side of the unity line than movements on the right side of the stimulus (triangles). This suggests that there may be a difference between the effect of the illusion on the left and the right hand depending on the side of the stimulus at which the movement is made. In order to find out whether this was so, we determined the effect of the illusion on movements separately for each side of the stimulus (left and right) and for each hand averaging over the large and medium movement types in both visibility conditions. Figure 4 shows that, except for subject 6, all subjects show a lateralisation effect on the left side of the stimulus that is opposite to the lateralisation
Fig. 2 Effect of the illusion on each movement type (movements in which a large, a medium or no effect of the illusion is expected) for the hand visible condition (a) and for the handinvisible condition (b). Light gray bars represent movements with the left hand and dark gray bars represent movements with the right hand. Error bars represent standard errors of the mean (between subjects)
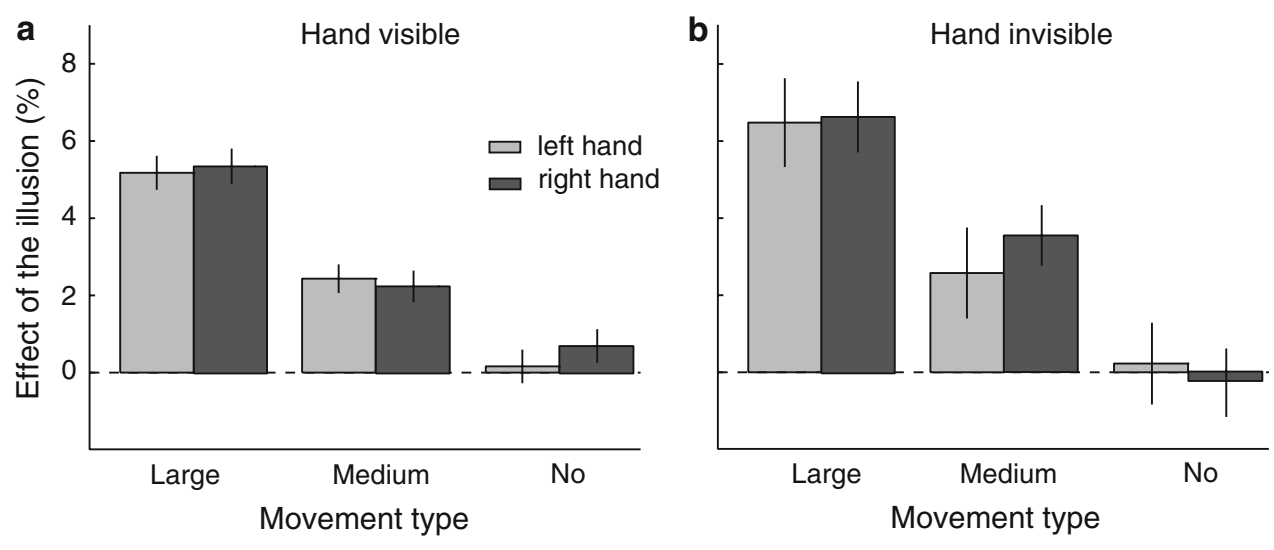


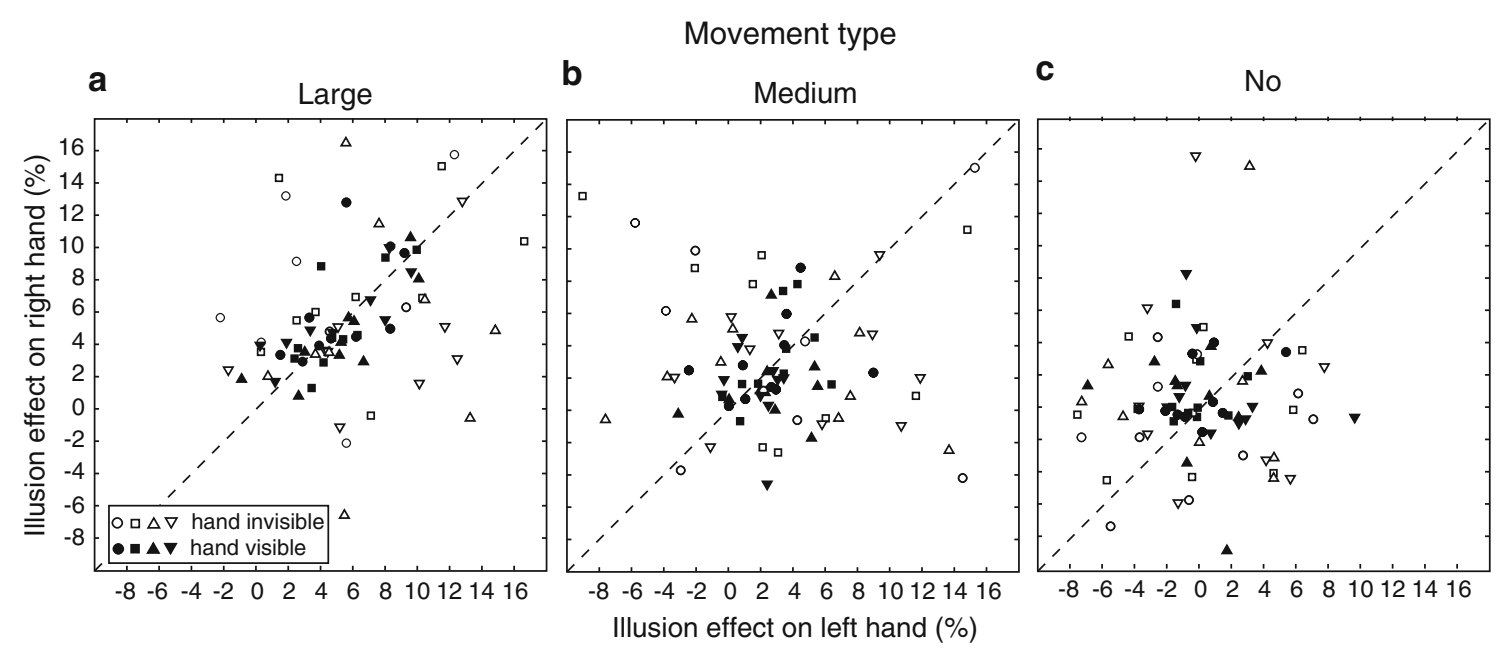

Fig. 3 The relationship between the influence of the illusion on the left and the right hand for each subject in the condition in which the hand was visible (filled symbols) and the condition in which the hand was invisible (open symbols). The different shapes of the symbols rep- resent the movement types (see Fig. 1d). Movements in which a large illusion effect is expected, are shown in (a). In (b) movements are shown in which a medium effect of the illusion is expected. Movements in which no illusion effect is expected are shown in (c)
Fig. 4 Effect of the illusion on each hand in each side of the stimulus (for each subject: averaged over movements on which a large and medium effect is expected)
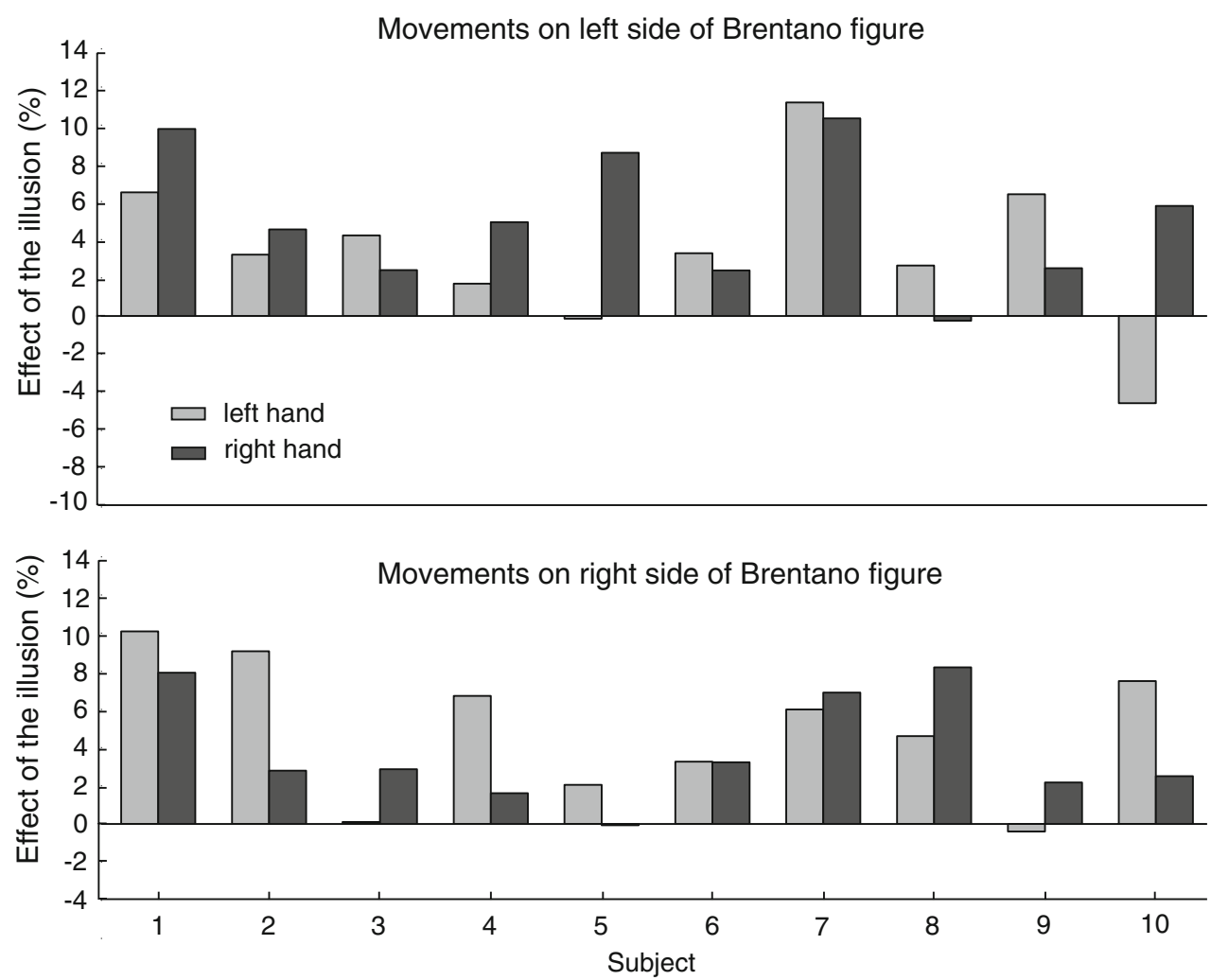

effect on the right side of the stimulus. Thus, there are differences between the left and right hand, but they depend on the subject and the location in the stimulus in which the movement is made.

As expected, the standard deviations in the movement amplitudes were larger in the hand-invisible condition $(0.99 \pm 0.12 \mathrm{~cm})$ than in the condition in which the hand was visible $(0.56 \pm 0.06 \mathrm{~cm})(F(1,9)=66.05, p<0.001)$.
Furthermore, the ANOVA showed a main effect of hand $(F(1,9)=18.87, p=0.001)$, with the left hand $(0.85 \pm$ $0.14 \mathrm{~cm})$ being slightly more variable than the right one $(0.70 \pm 0.09 \mathrm{~cm})$. The variability of the movements also depended on the type of movement $(F(1,9)=19.43$, $p<0.001)$, with the largest variability in movements in which no illusion effect was expected (between the middle arrowhead \& outside and between the outer arrowheads). 


\section{Discussion and conclusion}

In the study of Gonzalez et al. (2006) the Ponzo and Ebbinghaus illusion affected the peak grip aperture of the left hand more than that of the right hand. As we argued in the introduction, this could be due to a difference in proprioceptive accuracy between the hands. Following similar reasoning for our pointing study, we expected poorer proprioception to make one rely more on movement extent, which is influenced by the Brentano illusion. We therefore investigated whether a particularly large illusion effect would emerge for the left hand. If this had been so we would have found additional support for our interpretation. We expected a particularly large difference between the hands when we forced subjects to rely heavily on proprioceptive information for localizing their hand by removing visual feedback about the position of their hand. We did not find systematic differences in illusion effect between the hands, irrespective of whether the hand was visible or not. Movements were more variable when the hand was not visible, and the left hand was more variable than the right one, but the effect of the illusion was similar in all cases. The illusion effects for movements between the central and outer arrowheads (5-7\%) are similar to those of our previous study (de Grave et al. 2004), on which we based the methods. These effects are well below the magnitude of the effect of the illusion on length judgments (about 25\%; de Grave et al. 2004), so the failure to find a difference between the hands is unlikely to be due to a ceiling effect.

Although on average the left hand was not more susceptible to illusions than the right hand (Fig. 2), large differences between the left and the right hand did emerge when we looked at the illusion effects for individual subjects separately on each side of the stimulus. For half of the subjects a larger illusion effect on movements with the left hand was found in the right side of the stimulus, whereas the right hand was more affected in the left part of the stimulus and vice versa for four of the five other subjects (Fig. 4). Thus there are differences in the magnitude of the effect between the hands, but they are not consistent across subjects or space. Such differences probably outweigh any influence of differences in proprioception between the hands.

One explanation for not finding a consistent effect might be the task. In contrast to the grasping study of Gonzalez et al. (2006), we used a pointing task to avoid possible influences of responses to haptic feedback. In our view there is no a priori reason why grasping would be affected differently by the illusion than by pointing, as grasping can be considered as pointing with two digits (Smeets and Brenner 1999). Moreover, also Gonzalez et al. (2006) would not predict a difference in results due to the task, as pointing and grasping are both considered to be motor tasks, controlled by the dorsal stream. In line with our results, Radoeva et al. (2005) found no difference in effect of the Müller-Lyer illusion between grasping with the left and grasping with the right hand in their control subjects.

Instead of the difference in motor task, the difference in visual feedback of the stimulus might be the relevant factor. Bruno et al. (2008) suggested that visual feedback about the target is one of the important factors that determine the effect of illusions on pointing movements. In the study of Gonzalez et al. (2006) the target remained visible during the movement, whereas in this study the target was invisible during that period. One might argue that the removal of target information is the reason that we did not find a lateralisation of illusion effects. However, Gentilucci et al. (1997) did not find different effects of the Muller-Lyer illusion on pointing between the left and the right hand when the target was visible during the movement. In addition, the control subjects of Radoeva et al. (2005) showed no difference in effect of the Müller-Lyer illusion between the left and the right hand when grasping in the light. Except for the study of Gonzalez et al. (2006), all studies investigating illusion effects on the left and the right hand reported no difference. Thus there is no reason to assume that visibility of the target or the exact motor task is the critical difference between our study and that of Gonzalez et al. (2006).

Gonzalez et al. (2006) found a larger illusion effect on the peak grip aperture during precision grasping movements performed with the left hand, regardless of the subject's handedness. Milner and Goodale (2006) have argued that this finding challenges the idea of the presence of dissociation between position and extent, rather than between perception and action, as an explanation for the illusion effects on various tasks (Smeets and Brenner 1999, 2001; Smeets et al. 2002). As argued in the introduction, the results of Gonzalez et al. (2006) can easily be explained by the dissociation between position and extent. Contrary to all predictions (our prediction based on proprioceptive precision and that of lateralisation of precision grasping; Gonzalez et al. 2007), we found no difference in illusion effect between the left and the right hand. We tested one possible explanation of the lateralisation, but could not reject it because there simply was no effect to explain. We conclude that movements with the left and the right hand are equally susceptible to the Brentano illusion.

Open Access This article is distributed under the terms of the Creative Commons Attribution Noncommercial License which permits any noncommercial use, distribution, and reproduction in any medium, provided the original author(s) and source are credited.

\section{References}

Biegstraaten M, De Grave DDJ, Brenner E, Smeets JBJ (2007) Grasping the Müller-Lyer illusion: not a change in perceived length. Exp Brain Res 176:497-503 
Bruno N, Bernardis P, Gentilucci M (2008) Visually guided pointing, the Muller-Lyer illusion, and the functional interpretation of the dorsal-ventral split: conclusions from 33 independent studies. Neurosci Biobehav Rev 32:423-437

Cuijpers RH, Smeets JBJ, Brenner E (2004) On the relation between object shape and grasping kinematics. J Neurophys 91:2598-2606

Cuijpers RH, Brenner E, Smeets JBJ (2008) Consistent haptic feedback is required but not enough for natural reaching to virtual cylinders. Hum Mov Sci. doi:10.1016/j.humov.2008.07.003

De Grave DDJ, Brenner E, Smeets JBJ (2004) Illusions as a tool to study the coding of pointing movements. Exp Brain Res 155:56-62

De Grave DDJ, Biegstraaten M, Brenner E, Smeets JBJ (2005) Effects of the Ebbinghaus figure on grasping are not only due to misjudged size. Exp Brain Res 163:58-64

Gentilucci M, Daprati E, Gangitano M, Toni I (1997) Eye position tunes the contribution of allocentric and egocentric information to target localization in human goal-directed arm movements. Neurosci Lett 222:123-126

Goble DJ, Brown SH (2008) The biological and behavioral basis of upper limb asymmetries in sensorimotor performance. Neurosci Biobehav Rev 32:598-610

Gonzalez CLR, Ganel T, Goodale MA (2006) Hemispheric specialization for the visual control of action is independent of handedness. J Neurophys 95:3496-3501

Gonzalez CLR, Whitwell RL, Morrissey B, Ganel T, Goodale MA (2007) Left handedness does not extend to visually guided precision grasping. Exp Brain Res 182:275-279
Haffenden AM, Goodale MA (2000) Independent effects of pictorial displays on perception and action. Vis Res 40:1597-1607

Jeannerod M (1981) Intersegmental coordination during reaching at natural visual objects. In: Long J, Baddeley U (eds) Attention and performance IX. Erlbaum Hillsdale, New Jersey, pp 153-169

Milner D, Goodale MA (2006) The visual brain in action, 2nd edn. Oxford University Press, Oxford, pp 243-244

Oldfield RC (1971) The assessment and analysis of handedness: the Edinburgh inventory. Neuropsychologia 9:97-113

Radoeva PD, Cohen JD, Corballis PM, Lukovits TG, Koleva SG (2005) Hemispheric asymmetry in a dissociation between the visuomotor and visuoperceptual streams. Neuropsychologia 43:1763-1773

Smeets JBJ, Brenner E (1999) A new view on grasping. Motor Control 3:237-271

Smeets JBJ, Brenner E (2001) Independent movements of the digits in grasping. Exp Brain Res 139:92-100

Smeets JBJ, Brenner E, De Grave DDJ, Cuijpers RH (2002) Illusions in action: consequences of inconsistent processing of spatial attributes. Exp Brain Res 147:135-144

Stins JF, Kadar EE, Costall A (2001) A kinematic analysis of hand selection in a reaching task. Laterality 6:347-367

Van Beers RJ, Sittig AC, Denier van der Gon JJ (1998) The precision of proprioceptive position sense. Exp Brain Res 122:367-377

Van Beers RJ, Baraduc P, Wolpert DM (2002) Role of uncertainty in sensorimotor control. Phil Trans R Soc Lond B 357:1137-1145 